Brit. J. vener. Dis. (1961), 37, 176.

\title{
TREATMENT OF TRICHOMONAL VAGINITIS AND URETHRITIS AND THEIR COMPLICATIONS WITH METRONIDAZOLE (“FLAGYL")*
}

BY

P. POPCHRISTOV AND V. BEROV

Sofia, Bulgaria

Both the literature and personal investigations (Popchristov, 1957) indicate beyond any doubt that the relative percentage of urethritis and vaginitis due to Trichomonas vaginalis rises with increased rates of non-gonococcal genital infections. Of 278 patients with non-gonococcal urethritis and colpitis, and 102 patients with gonorrhoea seen at the Dispensary of the Institute for Skin and Venereal Diseases during the first six months of $1960, T$. vaginalis was proved to be the aetiological factor in 75 ( 27 per cent.).

An assumption exists that $T$. vaginalis is easily and commonly detected in females. As 40 per cent. of our patients suffering from trichomoniasis are males, we share the view that trichomoniasis is as common in men as in women. Increased attention to male cases of suspected trichomonal non-gonococcal urethritis and improved diagnostic methods have increased the number of male cases known to suffer from trichomoniasis.

In the past, the various treatments available were relatively inefficient and also inconvenient as their local and instrumental nature involved much waste of time for both patients and medical staff. Treatment was often uncompleted and recurrence, incomplete cure, and neurosis were encouraged. The past results of the oral treatment of trichomoniasis with arsenic, acridine, chinoline preparations, antibiotics, and sulphonamides were even less encouraging.

At the first Canadian Symposium on non-gonococcal urethritis and trichomoniasis (Montreal, September, 1959) the first observations were presented of a new synthetic compound (8823 R.P., "Flagyl"). Investigations carried out with this imidazole derivative by Cosar and Joulou (1959)

* Paper read at the European Symposium of the I.U.V.D.T. at Cracow, Poland, in September, 1960. stimulated Durel, Roiron, Siboulet, and Borel (1960) to conduct clinical trials which established its trichomonicidal effects. Studies in vitro and in vivo showed that it had practically no toxicity in therapeutic doses, wherein it differed substantially from the antitrichomonal drugs hitherto in use. It is a specific antitrichomonal preparation, and is inactive against Candida albicans and other aetiologic agents of non-gonococcal urethritis.

\section{Personal Observations}

As reports on the clinical application of "Flagyl" are still relatively few, we record our personal experience in the treatment of trichomoniasis with "Flagyl".

"Flagyl" is available as tablets of $0.250 \mathrm{~g}$. for oral administration and in tablets of $0.5 \mathrm{~g}$. for local vaginal application. In men, $0.250 \mathrm{~g}$. twice daily by mouth for 10 days is given. If required, the daily dose may be increased to $0.750 \mathrm{~g}$. or even to $1 \mathrm{~g}$., and treatment may be prolonged for more than 10 days or the original course may be repeated for another 10 days. In females, we use one tablet $(0.5$ g.) per vaginam each evening for 10 to 20 days, including the menstrual period.

A series of 22 males and females aged between 21 and 26 years, with trichomoniasis was treated with "Flagyl" between November, 1959, and July, 1960. In fourteen, the disorder was of 1 to 10 years' duration and in the other eight from 1 to 7 months. In all cases $T$. vaginalis was demonstrated in the urethral and vaginal discharge or in the prostate secretion, or on puncture of the epididymis. The parasites were detected in wet preparations, in Giemsa stained preparations, and in cultures (Pavlova's medium). 
Whether treated in the ward or as outpatients of the Institute for Skin and Venereal Diseases, the patients were examined each day for the presence of $T$. vaginalis in the vaginal and urethral discharges. Follow-up examinations with cultures were made for 3 to 7 months after the disappearance of the parasites. Prostatitis was present as a complication in four: in one of these $T$. vaginalis was discovered in the prostatic secretions; cystitis was present in another; and prostatitis and epididymitis occurred in one male in whom living $T$. vaginalis was found on puncture of the epididymis. The last is the first case of trichomonal epididymitis observed in Bulgaria.

In seventeen cases, examination of both partners was carried out. All female partners of infected males had clinical signs of trichomonal colpitis with parasites in the vaginal discharge. Two male partners of infected females had parasites in the urethra with no sign of urethritis and another had urethritis without $T$. vaginalis. In the rest of the men urethritis and $T$. vaginalis were both present. These observations support the view that trichomonal invasion in man is of a venereal character. They also indicate that successful treatment and prophylaxis of trichomoniasis requires the treatment of both partners even when the clinical and bacteriological findings in one of them are negative.

Analysis of our results shows that treatment of trichomoniasis with "Flagyl" leads to the rapid disappearance of $T$. vaginalis from the urethral and vaginal discharge and from the prostate and epididymis. Parasites disappeared in from 3 to 20 days (average 5 to 7). In some cases, mainly those of short duration and without complications, the discharge and subjective complaints disappeared at the same time as the parasites. In most cases, when the objective signs and parasites had disappeared, the urethra was dry. In one-third of patients, despite the disappearance of parasites, a moderate discharge still remained for a more or less continuous period, similar to post-gonococcal catarrh.

In two of our patients, after the definite disappearance of the parasites and a considerable diminution in the discharge, an increase in the discharge occurred with no signs of re-infection. We consider such cases as due to post-trichomonal catarrh. We are impressed by the rapid subsidence of objective signs in cases of prostatic and epididymal trichomoniasis treated with "Flagyl". The subsidence of cervical signs was noted (even when $T$. vaginalis had not been found in the cervical canal). When the objective signs do not improve or even become worse, either therapeutic failure or candidiasis is responsible. We have the impression that candidiasis may not only accompany trichomonal colpitis but may occur as a super-infection after treatment with "Flagyl".

Among our 22 cases we had only one relative failure. This woman was suffering from trichomonal vaginitis of 4 months' duration accompanied by candida colpitis, and she was given only local treatment for 10 days with $500 \mathrm{mg}$. "Flagyl" daily. Reinfection was excluded and no $T$. vaginalis was found in the urethra. The failure is attributed to the small dose and eventually to overlooked complications; her partner was cured in one course of oral treatment. A second course of "Flagyl" given both orally and locally one month later produced a complete clinical and bacteriological cure.

The doses of "Flagyl" for trichomoniasis recommended in the beginning of the present report are quite effective, and both local and oral treatment should be carried out simultaneously in females. This combination avoids the necessity of giving oral administration if the purely local treatment fails. In long-standing and complicated cases, when the parasites begin to disappear at the end of treatment, it is more rational to continue or to increase the daily dose.

The general tolerance to "Flagyl" was good, and in no case was treatment interrupted because of intolerance. Nevertheless, we have observed sideeffects in nine patients (one man and eight women). The symptoms subsided spontaneously in 1 or 2 days after the completion of treatment:

General lassitude ( 2 cases).

Headache (1 case).

Stomach disorder (1 case).

Nausea ( 2 cases).

Marked vaginal itching ( 3 cases).

General side-effects were observed only with oral treatment. The males seem to tolerate "Flagyl" better than the females. In the latter, marked itching of the vagina, labia, and even of the skin of the mons veneris and femoral regions may develop 3 to 4 days after the initiation of treatment. This may be due to the local application of the preparation. In our three patients who complained of pruritus, superimposed candidiasis was reported. Additional local treatment of the candidiasis was followed by the relief of the itching and disappearance of the candidiasis.

Apart from itching, the local application of "Flagyl" in our patients did not cause inflammation of the vagina or urethra. The vaginal tablets usually melt gradually and completely in 24 hours, but complete melting required 36 hours in one patient 
and 48 hours in another two. As a result we lengthened the interval between applications of tablets.

As "Flagyl" is a nitrogen compound, its effect upon the blood picture, especially in patients displaying general side-effects, was investigated. No change in the white or red blood picture, thrombocytes, or erythrocyte sedimentation rate, was noted. The spermograms, examined before, during, and after treatment with "Flagyl", were normal.

\section{Conclusion}

"Flagyl" deserves special attention and should find wide application in the treatment of trichomoniasis in both males and females because of its rapid effect and convenient modes of application.

\section{REFERENCES}

Cosar, C., and Julon, L. (1959). Ann. Inst. Pasteur, 96, 238.

Durel, P., Roiron, V., Siboulet, A., and Borel, L. J. (1960). Brit. J. vener. Dis., 36, 21.

Popchristov, P. (1957). "Urétrites non-gonococciques, medicine et culture physique". Sofia.

Traitement de la vaginite et de l'urétrite à Trichomonas Résumé

Le "Flagyl" retient l'attention et devrait être employé fréquemment dans le traitement des infections par le Trichomonas des hommes et des femmes à cause de la rapidité de son action et de la commodité de son administration. 